\section{Índice de conicidade como preditor de alterações no perfil lipídico em adolescentes de uma cidade do Nordeste do Brasil}

\author{
Conicity index as a predictor of changes in the \\ lipid profile of adolescents in a city in \\ Northeast Brazil
}

\author{
Índice de conicidad como predictor de \\ alteraciones en el perfil lipídico en adolescentes \\ de una ciudad del nordeste de Brasil
}

\section{Resumo}

O objetivo foi avaliar o índice de conicidade (Índice C) como preditor de alterações no perfil lipídico em adolescentes e estabelecer seus pontos de corte. Estudo transversal com 774 adolescentes de ambos os sexos (55\% do sexo feminino), de 10 a 14 anos de idade. O Índice $C$ foi calculado de acordo com a fórmula proposta por Valdez, considerando as medidas de massa corporal, estatura e circunferência da cintura (CC). As alterações no perfil lipídico dos adolescentes foram definidas segundo uma das seguintes condições: elevados níveis de colesterol total, lipoproteina de alta densidade (LDL), triglicerídeos e níveis baixos de lipoproteina de baixa densidade (HDL). O poder preditivo do Índice $C$ para alterações no perfil lipídico, assim como seus pontos de corte, foi determinado por meio das curvas ROC (Receiver Operating Characteristic). $O$ Índice $C$ foi um bom preditor para alterações lipídicas em adolescentes, com destaque para os níveis de triglicerídeos em adolescentes do sexo masculino de 10 a 11 anos (ROC =0,67; IC95\%: 0,50-0,85) e de 12 a 14 anos $(R O C=0,69 ;$ IC95\%: 0,59-0,80), assim como em adolescentes do sexo feminino de 10 a 11 anos $(R O C=0,65$; IC95\%: 0,50-0,79); e LDL em adolescentes do sexo feminino de 10 a 11 anos (ROC =0,70; IC95\%: 0,59-0,80) e adolescentes do sexo masculino ( $R O C=0,65$; IC95\%: 0,55-0,75) e adolescentes do sexo feminino ( $R O C=0,62$; IC95\%: 0,50-0,75) de 12 a 14 anos. Os pontos de corte do Índice $C$ variaram de 1,12 a 1,16 entre meninos e meninas. $O$ Índice $C$ pode ser utilizado para predizer alterações lipídicas, bem como seus pontos de corte podem ser utilizados para triagem de adolescentes em risco de alterações no perfil lipídico.

Dislipidemias; Obesidade Abdominal; Circunferência da Cintura; Adolescente
Adélia da Costa Pereira de Arruda Neta 1 José Cazuza de Farias Júnior 1 Pamela Rodrigues Martins 1

Flávia Emília Leite de Lima Ferreira 1

doi: 10.1590/0102-311X00029316

\author{
Correspondência \\ A. C. P. Arruda Neta \\ Universidade Federal da Paraíba. \\ Rua Rosa Lima dos Santos 90, João Pessoa, PB, \\ 58051-590, Brasil. \\ adeliacpereira@gmail.com \\ 1 Universidade Federal da Paraíba, João Pessoa, Brasil.
}




\section{Introdução}

Alterações nas concentrações dos lipídeos plasmáticos em adolescentes têm sido atribuídas especialmente ao aumento da prevalência de sobrepeso e obesidade ${ }^{1}$. A dislipidemia é apontada como um dos principais fatores de risco cardiovasculares, o que reforça a necessidade de um olhar diferenciado em adolescentes, cujo perfil do estado nutricional mudou bruscamente nos últimos 20 anos.

Estima-se que 20,5\% dos adolescentes do Brasil tenham excesso de peso corporal 2. Estudos têm consistentemente demonstrado uma associação de indicadores de sobrepeso e obesidade com alterações no perfil lipídico (dislipidemias) 3,4,5,6,7,8.

Dentre os indicadores antropométricos, utilizados para predizer alterações no perfil lipídico, o índice de massa corporal tem sido o mais utilizado em estudos populacionais com adolescentes. Entretanto, novos indicadores têm sido propostos com a finalidade de detectar a obesidade e a distribuição da gordura corporal como, por exemplo, o índice de conicidade (Índice C) 9 .

O Índice C é determinado com base na medida de massa corporal, estatura e circunferência abdominal, representando um indicador de obesidade abdominal, e parte do principio de que algumas pessoas acumulam gordura ao redor do abdômen, com a consequente alteração do desenho corporal da forma de um cilindro para um duplo-cone (dois cones com uma base comum), dispostos um sobre o outro, enquanto aquelas com menor quantidade de gordura na região central teriam aparência de um cilindro ${ }^{10}$. Este índice tem sido foco de diversos estudos com intuito de avaliar a sua possível relação com variáveis consideradas como de risco para o desenvolvimento de doenças cardiovasculares 11,12,13,14,15,16, tendo em vista que representa um bom indicador de obesidade, sobretudo central 17,18.

Embora exista associação entre excesso de peso corporal e os níveis lipídicos, poucos estudos 17 identificaram pontos de corte de indicadores antropométricos que visem à detecção de alterações no perfil lipídico em adolescentes.

Desse modo, o objetivo do presente estudo foi avaliar o Índice $\mathrm{C}$ como preditor de alterações no perfil lipídico em adolescentes e estabelecer seus pontos de corte.

\section{Métodos}

Estudo transversal baseado nos dados do primeiro ano (2014) do Estudo Longitudinal sobre Comportamento Sedentário, Atividade Física, Alimentação e Saúde dos Adolescentes (Estudo LONCAAFS). A proposta do LONCAAFS é realizar um acompanhamento dos adolescentes por um período de quatro anos, a fim de analisar as inter-relações entre o nível de atividade física, comportametnos sedentários, hábitos alimentares, qualidade de vida e indicadores de saúde de adolescentes, e de fatores psicossociais e ambientais com os níveis de prática de atividade física e comportamentos sedentários.

Para o cálculo de tamanho da amostra do Estudo LONCAAFS, consideraram-se os seguintes parâmetros: tamanho da população de referência igual a 9.520 adolescentes do 6 o ano do Ensino Fundamental II; prevalência do desfecho igual a 50\%, para captar o maior tamanho possível de amostra; intervalo de $95 \%$ de confiança; erro absoluto aceitável de 4 p.p. e efeito de desenho (deff) igual a dois. Com base nesses parâmetros, o tamanho mínimo da amostra original ficou estabelecido em 1.130 adolescentes, número que foi acrescido de $40 \%$ para compensar perdas e recusas, resultando em uma amostra final de 1.582 adolescentes.

Das 28 escolas selecionadas para compor a amostra, foram sorteadas 17 de forma aleatória, separadas por tipo (municipal e estadual) e distribuídas proporcionalmente de acordo com o tamanho (número de alunos matriculados no 6o ano) e por região geográfica (norte, sul, leste e oeste), para compor a subamostra. Optou-se por sortear apenas 17 escolas para compor a subamostra, por questões logísticas. A subamostra apresentou a mesma representatividade da população em relação à distribuição das escolas por tipo e região do município. A posteriori, foi realizado o cálculo do poder da subamostra para o presente estudo, que foi de $99 \%$.

A coleta foi realizada entre março e dezembro de 2014, por equipe composta por professores, estudantes de graduação e pós-graduação e profissionais das áreas de nutrição, educação física e enfermagem. A equipe foi devidamente treinada e recebeu manuais e protocolos, internos do estudo para realização da coleta de dados. 
Para avaliar o estado nutricional, foram coletadas medidas de peso, estatura e circunferência da cintura (CC). Essas medidas foram tomadas em triplicata, sempre pelo mesmo avaliador, e considerou-se sua média. Para medida de peso, foi utilizada uma balança digital, com precisão de 100 gramas, da marca Techline (São Paulo, Brasil). Para efetuar a medida de estatura foi utilizado um estadiômetro portátil da marca Sanny (São Bernardo do Campo, Brasil). Uma fita métrica inelástica da marca Sanny foi utilizada para mensurar a CC dos adolescentes. A CC foi mensurada com uma fita antropométrica no ponto médio entre a última costela e a crista ilíaca.

O Índice C foi determinado por meio das medidas de peso, estatura e CC conforme a seguinte equação matemática 10:

$$
\text { Índice } C=\frac{\text { Circunferência da cintura }(\mathrm{m})}{0,109 \sqrt{\frac{\text { Peso corporal }(\mathrm{kg})}{\text { Estatura }(\mathrm{m})}}}
$$

O valor 0,109 é a constante que resulta da raiz da razão entre $4 \pi$ (advindo da dedução do perímetro do círculo de um cilindro) e a densidade média do ser humano de $1,050 \mathrm{~kg} / \mathrm{m}^{3}$. Sua principal ideia é que pessoas com menor acúmulo de gordura na região central teriam a forma corporal semelhante à de um cilindro e aqueles com maior acúmulo teriam a semelhança com um duplo cone, tendo uma base em comum, dispostos um sobre o outro 10.

No presente estudo, optou-se por realizar a análise do Índice C para a predição das alterações lipídicas separadamente para adolescentes dos sexos masculino e feminino, uma vez que o comportamento dos lipídios difere entre os sexos por causa dos hormônios sexuais endógenos ${ }^{17}$. Além disso, a amostra foi dividida em grupos de idade que, de acordo com a literatura ${ }^{19}$, representam o início (10-11 anos) e a transição (12-14 anos) para a segunda etapa da adolescência.

Para a avaliação do perfil lipídico, os adolescentes foram submetidos a uma coleta de sangue da veia anticubital, após 12 horas de jejum. Minutos antes da coleta, era realizada uma anamnese com os adolescentes para identificar fatores que poderiam interferir nos resultados das análises bioquímicas (uso de medicamentos, alergias, quebra do jejum de 10 a 12 horas, realização de atividade física vigorosa). O sistema de coleta de sangue foi realizado com tubo a vácuo por técnicas de enfermagem com plena experiência em coleta sanguínea.

As concentrações de triglicerídeos, colesterol total e colesterol de alta densidade (HDL) foram determinadas pelo método de turbidimetria, recorrendo-se ao analisador bioquímico automático Labmax 240 premium (Labtest, Lagoa Santa, Brasil). O colesterol de baixa densidade (LDL) foi determinado pela equação de Friedwald, considerando os valores de concentração do colesterol HDL, VLDL e colesterol total ([colesterol total-colesterol HDL]-[triglicerídeos/5]). Neste estudo, para classificar os marcadores do perfil lipídico em normal e alterado, foram utilizados como referência os pontos de corte para crianças e adolescentes, contidos na V Diretriz Brasileira de Dislipidemias e Prevenção da Aterosclerose (2013) 20; para hipertrigliceridemia e LDL alterado, foram considerados como alterados valores de LDL-c iguais ou acima de $130 \mathrm{mg} / \mathrm{dL}$; para hipercolesterolemia, foram considerados valores do colesterol total iguais ou acima de $170 \mathrm{mg} / \mathrm{dL}$; e para hipoalfalipoproteinemia, foram considerados valores de HDL-c iguais ou inferiores a $45 \mathrm{mg} / \mathrm{dL}$.

Para descrever as características da amostra, foram utilizados os procedimentos da estatística descritiva, incluindo média e desvio-padrão para as variáveis idade, peso, estatura, CC, índice de massa corporal e Índice C. Para comparar os valores médios dessas variáveis entre o grupo de adolescentes dos sexos masculino e feminino, foi utilizado o teste $t$ de Student. Foi adotado nível de significância de $5 \%$ para todos os testes.

O poder preditivo do Índice $\mathrm{C}$ para alterações lipídicas foi avaliado por meio das curvas Receiver Operating Characteristic (ROC) 21. Inicialmente, foi identificada a área total sob a curva ROC entre o Índice $\mathrm{C}$ e os marcadores de alterações lipídicas. Quanto maior a área sob a curva ROC, maior o poder preditivo do Índice $\mathrm{C}$ para alterações lipídicas. Para identificar a diferença das áreas sob as curvas ROC, entre os grupos de idades e sexo, aplicou-se o teste do qui-quadrado. Na sequência, foram identificados os pontos de corte para o Índice $\mathrm{C}$ que obteve áreas significativas sob a curva ROC, com seus respectivos valores de sensibilidade e especificidade. Considerou-se como critério para obter os pontos de corte do Índice C como preditor de alterações lipídicas os valores com sensibilidade e especificidade mais equilibrados entre si. 
Na curva ROC, um preditor perfeito corresponderia a uma linha horizontal no topo do gráfico, porém essa linha horizontal é dificilmente alcançada. Na prática, curvas consideradas boas estarão entre a linha diagonal e a linha perfeita, em que quanto maior a distância da linha diagonal, melhor o sistema 22 . Portanto, neste estudo, o Índice $\mathrm{C}$ foi considerado bom preditor quando seu intervalo de confiança (IC) for superior a 0,50 e a área sob a curva ROC for superior a 0,60. O IC determina se a capacidade preditiva do indicador antropométrico não é devido ao acaso e o seu limite inferior não deve ser menor que 0,50 23. Neste estudo, utilizou-se um intervalo com 95\% de confiança (IC95\%).

O número de pontos de corte escolhidos na curva ROC varia de acordo com a necessidade do pesquisador 24 . O ponto de corte é definido com um valor que pode ser selecionado arbitrariamente pelo pesquisador entre os valores possíveis para a predição 22 . Neste estudo, para discriminar a hipertrigliceridemia, o LDL-c alterado, a hipoalfalipoproteinemia e a hipercolesterolemia, escolheu-se utilizar o ponto de corte mais baixo, em cada faixa etária e de acordo com o sexo.

Os critérios de exclusão adotados foram: adolescentes fora da faixa etária de interesse do estudo (abaixo de 10 e acima de 14 anos); não ter seguido os procedimentos adequados de preparação para a sua realização do exame de sangue (jejum) e/ou não ter realizado medidas antropométricas (estatura, massa corporal, circunferência abdominal).

Os dados foram tabulados no EpiData 3.1 (Epidata Assoc., Odense, Dinamarca), seguindo um processo de dupla digitação, com checagem automática de consistência das respostas das variáveis. A ferramenta "validar dupla digitação" foi utilizada para identificar possíveis erros de digitação. Todos os erros foram identificados e corrigidos conforme os valores originais dos questionários. Todas as análises foram realizadas no Stata 13.0 (StataCorp LP, College Station, Estados Unidos).

O projeto foi aprovado pelo Comitê de Ética em Pesquisas com Seres Humanos do Centro de Ciências da Saúde da Universidade Federal da Paraíba (registro no 15268213.0.0000.5188) e cada entrevista foi conduzida somente após o esclarecimento dos objetivos da pesquisa e consentimento do participante, mediante assinatura do Termo de Consentimento Livre e Esclarecido.

\section{Resultados}

A amostra foi composta por 774 adolescentes, sendo 55\% composta por adolescentes do sexo feminino (423) com idade média de 11,77 anos $( \pm 0,86)$. Houve diferença significativa, entre adolescentes dos sexos masculino e feminino para a idade e o Índice C $(\mathrm{p}<0,05)$. As demais características da amostra não tiveram diferenças significativas entre os sexos, como pode ser visto na Tabela 1.

A Tabela 2 apresenta o perfil lipídico da amostra estudada segundo o sexo, e sua classificação (normal e alterado). Apesar de os adolescentes do sexo feminino apresentarem um perfil lipídico ligeiramente mais alterado do que os adolescentes do sexo masculino, a diferença não foi estatisticamente significante.

Os valores da área sob a curva ROC, para o Índice C como preditor de alterações no perfil lipídico, assim como os pontos de corte, a sensibilidade e especificidade são apresentados na Tabela 3.

No presente estudo, o Índice $\mathrm{C}$ se mostrou um bom preditor para hipercolesterolemia apenas em adolescentes do sexo masculino de 12 a 14 anos (ROC = 0,63; IC95\%: 0,56-0,70) e em adolescentes do sexo feminino de 10 a 11 anos (ROC = 0,61; IC95\%: 0,52-0,71). Quanto à hipoalfalipoproteinemia, o Índice C não foi um bom preditor para adolescentes do sexo masculino (ROC = 0,58; IC95\%: 0,510,65) nem para adolescentes do sexo feminino (ROC = 0,60; IC95\%: 0,52-0,67), ambos com idades de 12 a 14 anos.

Para predição de hipertrigliceridemia, o Índice $\mathrm{C}$ foi bom preditor em adolescentes do sexo masculino de 10 a 11 anos (ROC = 0,67; IC95\%: 0,50-0,85) e de 12 a 14 anos (ROC = 0,69; IC95\%: 0,590,80), assim como em adolescentes do sexo feminino de 10 a 11 anos (ROC = 0,65; IC95\%: 0,50-0,79). $\mathrm{O}$ Índice $\mathrm{C}$ também foi um bom preditor para LDL-alterado em adolescentes do sexo masculino de 12 a 14 anos $(\mathrm{ROC}=0,65$; IC95\%: 0,55-0,75) e em adolescentes do sexo feminino de 10 a 11 anos $($ ROC = 0,70; IC95\%: 0,59-0,80) e de 12 a 14 anos (ROC = 0,62; IC95\%: 0,50-0,75).

Quanto aos pontos de corte do Índice C para triagem de alterações lipídicas, ficou estabelecido para meninos de 10 a 11 anos o ponto de corte de 1,16 (sensibilidade 63,27\% e especificidade 63,16\%) e para meninos de 12 a 14 anos o ponto de corte estabelecido foi de 1,14 (sensibilidade 58,65\% e 


\section{Tabela 1}

Características de adolescentes de 10 a 14 anos de João Pessoa, Paraíba, Brasil, 2014.

\begin{tabular}{lccc}
\hline Variáveis & $\begin{array}{c}\text { Masculino }(\mathbf{n}=\mathbf{3 5 1}) \\
\overline{\mathbf{X}}(\mathbf{D P})\end{array}$ & $\begin{array}{c}\text { Feminino }(\mathbf{n}=\mathbf{4 2 3}) \\
\overline{\mathbf{X}}(\mathbf{D P})\end{array}$ & $\begin{array}{c}\text { Valor de } \mathbf{p} \\
\text { Idade }\end{array}$ \\
Massa corporal & $12,19( \pm 1,01)$ & $11,77( \pm 0,86)$ & 0,03 \\
Estatura & $44,37( \pm 12,0)$ & $44,07( \pm 10,40)$ & 0,40 \\
IMC & $149,9( \pm 9,53)$ & $149,10( \pm 7,69)$ & 0,31 \\
CC & $19,45( \pm 4,36)$ & $19,50( \pm 4,26)$ & 0,56 \\
Índice C & $68,14( \pm 10,92)$ & $67,25( \pm 9,12)$ & 0,36 \\
\hline
\end{tabular}

CC: circunferência da cintura; DP: desvio padrão; IMC: índice de massa corporal; Índice C: Índice de Conicidade; X: média.

\section{Tabela 2}

Perfil lipídico de adolescentes de 10 a 14 anos de João Pesssoa, Paraíba, Brasil, 2014.

\begin{tabular}{|c|c|c|c|}
\hline & $\begin{array}{c}\text { Masculino }(\mathrm{n}=351) \\
\mathrm{n}(\%)\end{array}$ & $\begin{array}{c}\text { Feminino }(n=423) \\
n(\%)\end{array}$ & Valor de $p$ \\
\hline \multicolumn{4}{|l|}{ Colesterol total } \\
\hline Alterado * & $113(32)$ & $138(33)$ & 1,11 \\
\hline Normal & $238(68)$ & $285(67)$ & \\
\hline \multicolumn{4}{|l|}{ HDL-C } \\
\hline Alterado ** & $203(58)$ & $246(58)$ & 0,34 \\
\hline Normal & $148(42)$ & $177(42)$ & \\
\hline \multicolumn{4}{|l|}{ LDL-C } \\
\hline Alterado $* * \star$ & $46(13)$ & $44(10)$ & 0,97 \\
\hline Normal & 305 (87) & $379(90)$ & \\
\hline \multicolumn{4}{|l|}{ Triglicerídeos } \\
\hline Alterado \# & $76(22)$ & $100(24)$ & 1,77 \\
\hline Normal & $275(78)$ & $323(66)$ & \\
\hline
\end{tabular}

HDL: colesterol de alta densidade; LDL: colesterol de baixa densidade.

* Alterado > 170mg/dL;

** Alterado $<45 \mathrm{mg} / \mathrm{dL}$;

*** Alterado $>130 \mathrm{mg} / \mathrm{dL}$;

\# Alterado $>130 \mathrm{mg} / \mathrm{dL}$.

especificidade 58,71\%). Em meninas, o ponto de corte estabelecido foi de 1,14 (sensibilidade 52,53\% e especificidade 52,5\%) para faixa etária de 10 a 11 anos e de 1,12 (sensibilidade $58,16 \%$ e especificidade $58,89 \%)$ para faixa etária de 12 a 14 anos.

Na Tabela 4 está exposto o denominador do Índice C já calculado para adolescentes que apresentem peso entre $30 \mathrm{~kg}$ e $60 \mathrm{~kg}$ e estatura entre $1,40 \mathrm{~m}$ e $1,57 \mathrm{~m}$. 


\section{Tabela 3}

Área sob a curva ROC * (Receiver Operating Characteristic) e intervalo de 95\% de confiança (IC95\%), ponto de corte, sensibilidade e especificidade do Índice de Conicidade (Índice C) como predição de alterações no perfil lipídico de adolescentes de João Pessoa, Paraíba, Brasil, 2014.

\begin{tabular}{|c|c|c|c|c|}
\hline & \multicolumn{4}{|c|}{ Índice C } \\
\hline & Curva ROC (IC95\%) & Ponto de corte & Sensibilidade & Especificidade \\
\hline \multicolumn{5}{|c|}{ Masculino (10 a 11 anos de idade) } \\
\hline Colesterol total & $0,59(0,46-0,72)$ & 1,16 & $53,57 \%$ & $54,24 \%$ \\
\hline $\mathrm{HDL}$ & $0,60(0,48-0,73)$ & 1,16 & $63,27 \%$ & $63,16 \%$ \\
\hline LDL & $0,63(0,48-0,78)$ & 1,17 & $62,50 \%$ & $60,56 \%$ \\
\hline Triglicerídeos & $0,67(0,50-0,85)$ & 1,19 & $66,67 \%$ & $66,25 \%$ \\
\hline \multicolumn{5}{|c|}{ Masculino (12 a 14 anos de idade) } \\
\hline Colesterol total & $0,63(0,56-0,71)$ & 1,14 & $58,54 \%$ & $58,71 \%$ \\
\hline $\mathrm{HDL}$ & $0,58(0,51-0,65)$ & 1,14 & $56,43 \%$ & $56,70 \%$ \\
\hline LDL & $0,65(0,55-0,75)$ & 1,15 & $60,71 \%$ & $60,77 \%$ \\
\hline Triglicerídeos & $0,69(0,59-0,80)$ & 1,15 & $59,26 \%$ & $59,52 \%$ \\
\hline \multicolumn{5}{|c|}{ Feminino (10 a 11 anos de idade) } \\
\hline Colesterol total & $0,61(0,52-0,71)$ & 1,15 & $60,71 \%$ & $60,98 \%$ \\
\hline $\mathrm{HDL}$ & $0,56(0,47-0,64)$ & 1,14 & $52,53 \%$ & $52,50 \%$ \\
\hline LDL & $0,70(0,59-0,80)$ & 1,15 & $60,00 \%$ & $59,75 \%$ \\
\hline Triglicerídeos & $0,65(0,50-0,79)$ & 1,15 & $63,64 \%$ & $61,78 \%$ \\
\hline \multicolumn{5}{|c|}{ Feminino (12 a 14 anos de idade) } \\
\hline Colesterol total & $0,53(0,45-0,61)$ & 1,13 & $54,55 \%$ & $54,55 \%$ \\
\hline $\mathrm{HDL}$ & $0,60(0,52-0,67)$ & 1,12 & $58,16 \%$ & $58,89 \%$ \\
\hline LDL & $0,62(0,50-0,75)$ & 1,14 & $56,52 \%$ & $56,65 \%$ \\
\hline Triglicerídeos & $0,59(0,46-0,71)$ & 1,15 & $61,54 \%$ & $61,46 \%$ \\
\hline
\end{tabular}

HDL: colesterol de alta densidade; LDL: colesterol de baixa densidade.

* Área sob a curva ROC que demonstra poder discriminatório para alterações lipídicas (limite inferior do IC95\% > 0,50).

\section{Discussão}

O Índice C é considerado um bom discriminador de obesidade central e vem sendo relacionado a fatores de risco metabólicos e cardiovasculares em crianças, adolescentes e adultos 25,26,27,28,29. A incorporação da massa corporal e da estatura na fórmula do cálculo do Índice C o qualifica como um indicador de distribuição mais abrangente porque leva em conta o total de massa corporal 30. Este estudo é o primeiro no Brasil a publicar pontos de corte do Índice $\mathrm{C}$ para predizer alterações no perfil lipídico, em adolescentes, estratificados por sexo e idade.

A hipercolesterolemia, em crianças e adolescentes, está associada a dislipidemias na fase adulta 31 e à presença de aterosclerose subclínica ${ }^{32}$. Neste estudo, a prevalência de hipercolesterolemia foi de aproximadamente $32 \%$, confirmando uma tendência encontrada em outras pesquisas realizadas no Brasil em que a prevalência de hipercolesterolemia varia entre $10 \%$ a 35\%, a depender da região estudada 33,34 .

Recentemente, um estudo revelou que aproximadamente $20 \%$ dos adolescentes dos Estados Unidos apresentaram alguma alteração lipídica 35 . No presente estudo, percentual semelhante foi encontrado para hipertrigliceridemia (22\%), que é considerado um fator de risco cardiovascular e pode decorrer de fatores genéticos ou adquiridos como, por exemplo, a obesidade 36. Entretanto, tal valor foi muito superior ao encontrado em um estudo nacional, de base escolar, conduzido em 2013-2014, que encontrou valores entre 6,8\% (Região Norte) e 9,6\% (Região Nordeste) 37.

A prevalência de hipoalfalipoproteinemia, neste estudo, chegou a $58 \%$, resultado semelhante ao encontrado em um estudo realizado em Recife, Pernambuco (56\%; IC95\%: 51,3-60,5) 38. Um perfil lipídico marcado pela combinação da hipoalfalipoproteinemia e hipertrigleridemia costuma estar 
Tabela 4

Valor do denominador segundo peso e estatura para cálculo do Índice de Conicidade (Índice C) em adolescentes.

\begin{tabular}{|c|c|c|c|c|c|c|c|c|c|c|c|c|c|c|c|c|c|c|}
\hline$P(k g) / E(m)$ & 1,40 & 1,41 & 1,42 & 1,43 & 1,44 & 1,45 & 1,46 & 1,47 & 1,48 & 1,49 & 1,50 & 1,51 & 1,52 & 1,53 & 1,54 & 1,55 & 1,56 & 1,57 \\
\hline 30 & 0,50 & 0,50 & 0,50 & 0,50 & 0,50 & 0,50 & 0,49 & 0,49 & 0,49 & 0,49 & 0,49 & 0,49 & 0,48 & 0,48 & 0,48 & 0,48 & 0,48 & 0,48 \\
\hline 31 & 0,52 & 0,52 & 0,52 & 0,52 & 0,51 & 0,51 & 0,51 & 0,51 & 0,51 & 0,51 & 0,50 & 0,50 & 0,50 & 0,50 & 0,50 & 0,50 & 0,49 & 0,49 \\
\hline 32 & 0,52 & 0,52 & 0,52 & 0,52 & 0,51 & 0,51 & 0,51 & 0,51 & 0,51 & 0,51 & 0,50 & 0,50 & 0,50 & 0,50 & 0,50 & 0,50 & 0,49 & 0,49 \\
\hline 33 & 0,53 & 0,53 & 0,53 & 0,52 & 0,52 & 0,52 & 0,52 & 0,52 & 0,51 & 0,51 & 0,51 & 0,51 & 0,51 & 0,51 & 0,50 & 0,50 & 0,50 & 0,50 \\
\hline 34 & 0,54 & 0,54 & 0,53 & 0,53 & 0,53 & 0,53 & 0,53 & 0,52 & 0,52 & 0,52 & 0,52 & 0,52 & 0,52 & 0,51 & 0,51 & 0,51 & 0,51 & 0,51 \\
\hline 35 & 0,55 & 0,54 & 0,54 & 0,54 & 0,54 & 0,54 & 0,53 & 0,53 & 0,53 & 0,53 & 0,53 & 0,52 & 0,52 & 0,52 & 0,52 & 0,52 & 0,52 & 0,51 \\
\hline 36 & 0,55 & 0,55 & 0,55 & 0,55 & 0,55 & 0,54 & 0,54 & 0,54 & 0,54 & 0,54 & 0,53 & 0,53 & 0,53 & 0,53 & 0,53 & 0,53 & 0,52 & 0,52 \\
\hline 37 & 0,56 & 0,56 & 0,56 & 0,55 & 0,55 & 0,55 & 0,55 & 0,55 & 0,55 & 0,54 & 0,54 & 0,54 & 0,54 & 0,54 & 0,53 & 0,53 & 0,53 & 0,53 \\
\hline 38 & 0,57 & 0,57 & 0,56 & 0,56 & 0,56 & 0,56 & 0,56 & 0,55 & 0,55 & 0,55 & 0,55 & 0,55 & 0,55 & 0,54 & 0,54 & 0,54 & 0,54 & 0,54 \\
\hline 39 & 0,58 & 0,57 & 0,57 & 0,57 & 0,57 & 0,57 & 0,56 & 0,56 & 0,56 & 0,56 & 0,56 & 0,55 & 0,55 & 0,55 & 0,55 & 0,55 & 0,55 & 0,54 \\
\hline 40 & 0,58 & 0,58 & 0,58 & 0,58 & 0,57 & 0,57 & 0,57 & 0,57 & 0,57 & 0,56 & 0,56 & 0,56 & 0,56 & 0,56 & 0,56 & 0,55 & 0,55 & 0,55 \\
\hline 41 & 0,59 & 0,59 & 0,59 & 0,58 & 0,58 & 0,58 & 0,58 & 0,58 & 0,57 & 0,57 & 0,57 & 0,57 & 0,57 & 0,56 & 0,56 & 0,56 & 0,56 & 0,56 \\
\hline 42 & 0,60 & 0,59 & 0,59 & 0,59 & 0,59 & 0,59 & 0,58 & 0,58 & 0,58 & 0,58 & 0,58 & 0,57 & 0,57 & 0,57 & 0,57 & 0,57 & 0,57 & 0,56 \\
\hline 43 & 0,60 & 0,60 & 0,60 & 0,60 & 0,60 & 0,59 & 0,59 & 0,59 & 0,59 & 0,59 & 0,58 & 0,58 & 0,58 & 0,58 & 0,58 & 0,57 & 0,57 & 0,57 \\
\hline 44 & 0,61 & 0,61 & 0,61 & 0,60 & 0,60 & 0,60 & 0,60 & 0,60 & 0,59 & 0,59 & 0,59 & 0,59 & 0,59 & 0,58 & 0,58 & 0,58 & 0,58 & 0,58 \\
\hline 45 & 0,62 & 0,62 & 0,61 & 0,61 & 0,61 & 0,61 & 0,61 & 0,60 & 0,60 & 0,60 & 0,60 & 0,60 & 0,59 & 0,59 & 0,59 & 0,59 & 0,59 & 0,58 \\
\hline 46 & 0,62 & 0,62 & 0,62 & 0,62 & 0,62 & 0,61 & 0,61 & 0,61 & 0,61 & 0,61 & 0,60 & 0,60 & 0,60 & 0,60 & 0,60 & 0,59 & 0,59 & 0,59 \\
\hline 47 & 0,63 & 0,63 & 0,63 & 0,62 & 0,62 & 0,62 & 0,62 & 0,62 & 0,61 & 0,61 & 0,61 & 0,61 & 0,61 & 0,60 & 0,60 & 0,60 & 0,60 & 0,60 \\
\hline 48 & 0,64 & 0,64 & 0,63 & 0,63 & 0,63 & 0,63 & 0,62 & 0,62 & 0,62 & 0,62 & 0,62 & 0,61 & 0,61 & 0,61 & 0,61 & 0,61 & 0,60 & 0,60 \\
\hline 49 & 0,64 & 0,64 & 0,64 & 0,64 & 0,64 & 0,63 & 0,63 & 0,63 & 0,63 & 0,63 & 0,62 & 0,62 & 0,62 & 0,62 & 0,61 & 0,61 & 0,61 & 0,61 \\
\hline 50 & 0,65 & 0,65 & 0,65 & 0,64 & 0,64 & 0,64 & 0,64 & 0,64 & 0,63 & 0,63 & 0,63 & 0,63 & 0,63 & 0,62 & 0,62 & 0,62 & 0,62 & 0,62 \\
\hline 51 & 0,66 & 0,66 & 0,65 & 0,65 & 0,65 & 0,65 & 0,64 & 0,64 & 0,64 & 0,64 & 0,64 & 0,63 & 0,63 & 0,63 & 0,63 & 0,63 & 0,62 & 0,62 \\
\hline 52 & 0,66 & 0,66 & 0,66 & 0,66 & 0,66 & 0,65 & 0,65 & 0,65 & 0,65 & 0,64 & 0,64 & 0,64 & 0,64 & 0,64 & 0,63 & 0,63 & 0,63 & 0,63 \\
\hline 53 & 0,67 & 0,67 & 0,67 & 0,66 & 0,66 & 0,66 & 0,66 & 0,65 & 0,65 & 0,65 & 0,65 & 0,65 & 0,64 & 0,64 & 0,64 & 0,64 & 0,64 & 0,63 \\
\hline 54 & 0,68 & 0,67 & 0,67 & 0,67 & 0,67 & 0,67 & 0,66 & 0,66 & 0,66 & 0,66 & 0,65 & 0,65 & 0,65 & 0,65 & 0,65 & 0,64 & 0,64 & 0,64 \\
\hline 55 & 0,68 & 0,68 & 0,68 & 0,68 & 0,67 & 0,67 & 0,67 & 0,67 & 0,66 & 0,66 & 0,66 & 0,66 & 0,66 & 0,65 & 0,65 & 0,65 & 0,65 & 0,65 \\
\hline 56 & 0,69 & 0,69 & 0,68 & 0,68 & 0,68 & 0,68 & 0,68 & 0,67 & 0,67 & 0,67 & 0,67 & 0,66 & 0,66 & 0,66 & 0,66 & 0,66 & 0,65 & 0,65 \\
\hline 57 & 0,70 & 0,69 & 0,69 & 0,69 & 0,69 & 0,68 & 0,68 & 0,68 & 0,68 & 0,67 & 0,67 & 0,67 & 0,67 & 0,67 & 0,66 & 0,66 & 0,66 & 0,66 \\
\hline 58 & 0,70 & 0,70 & 0,70 & 0,69 & 0,69 & 0,69 & 0,69 & 0,68 & 0,68 & 0,68 & 0,68 & 0,68 & 0,67 & 0,67 & 0,67 & 0,67 & 0,66 & 0,66 \\
\hline 59 & 0,71 & 0,71 & 0,70 & 0,70 & 0,70 & 0,70 & 0,69 & 0,69 & 0,69 & 0,69 & 0,68 & 0,68 & 0,68 & 0,68 & 0,67 & 0,67 & 0,67 & 0,67 \\
\hline 60 & 0,70 & 0,71 & 0,71 & 0,71 & 0,70 & 0,70 & 0,70 & 0,70 & 0,69 & 0,69 & 0,69 & 0,69 & 0,68 & 0,68 & 0,68 & 0,68 & 0,68 & 0,67 \\
\hline
\end{tabular}

$\mathrm{E}(\mathrm{m})$ : estatura em metros; $\mathrm{P}(\mathrm{kg})$ : peso em $\mathrm{kg}$.

Nota: para identificar o valor do denominador do Índice C (cruzar peso e estatura na tabela). Depois, basta dividir o valor da circunferência da cintura (em metros) pelo denominador encontrado na tabela.

presente na obesidade 39 . Tal combinação também pode estar associada a pouca alteração dos níveis de LDL-c, assim como foi encontrado neste estudo, que já é descrito como um padrão na infância 40 .

$\mathrm{O}$ resultado obtido pelo Índice $\mathrm{C}$ mostra que a obesidade central pode estar relacionada com um perfil lipídico plasmático desfavorável à saúde ${ }^{41}$, com destaque para alterações nos níveis de triglicerídeos e LDL-c. Um estudo realizado com 127 crianças e adolescentes de 9 a 17 anos de idade constatou que quanto maior a quantidade de gordura acumulada na região central do corpo, maior era a alteração desses lipídeos 42 .

$\mathrm{O}$ Índice $\mathrm{C}$ foi um bom preditor para hipercolesterolemia em meninos de 12 a 14 anos e em meninas de 11 a 12 anos. Já para a hipoalfalipoproteinemia, o Índice $\mathrm{C}$ foi um bom preditor apenas para meninas de 12 a 14 anos, sendo este resultado semelhante ao de um estudo realizado com adolescentes de 14 a 19 anos, que teve o Índice C como bom preditor para hipoalfalipoproteinemia (HDL-baixo) apenas para meninas 17 .

Apesar de, neste estudo, o Índice C ter sido um bom preditor para hipertrigliceridemia e LDL-alterado, em meninos e meninas de algumas faixas etárias, não foram encontrados estudos que ava- 
liassem o poder preditivo de indicadores antropométricos, incluindo o Índice C, para alterações nos níveis de triglicerídeos e LDL em adolescentes, o que dificultou a comparação.

Neste estudo, houve uma diferença significativa $(\mathrm{p}=0,02)$ entre as médias do Índice $\mathrm{C}$, e os meninos apresentaram uma maior média $(1,16 \pm 0,07)$. Esse resultado pode estar associado ao fato de que adolescentes do sexo masculino, em geral, apresentam tendência a acumular uma mario quantidade de gordura na região abdominal 43,44,45. Mesmo demonstrando uma redução da gordura corporal nessa faixa etária 46,47, em adolescentes do sexo masculino, estima-se que valores plenos de massa livre de gordura sejam atingidos dois a três anos mais tarde que as meninas 47,48.

A gordura abdominal tende a se acumular em maior proporção que a gordura subcutânea ainda na infância em ambos os sexos. Contudo, a gordura abdominal apresenta uma tendência de aumento nos adolescentes do sexo masculino a partir dos 13 ou 14 anos de idade, o que pode explicar, em parte, o fato de a gordura nos meninos ser mais centralizada 35 . Além disso, esse fato pode justificar a discreta redução que tende a ocorrer nos níveis de colesterol total, HDL e LDL no início da adolescência e o retorno desses níveis, aos valores anteriores, no fim dessa primeira fase da vida 49.

Tais alterações citadas podem justificar o fato de o Índice C não conseguir predizer alterações no colesterol total, HDL e LDL na faixa de 10 a 11 anos em adolescentes do sexo masculino, que caracteriza o início da adolescência, porém conseguiu predizer todas as alterações lipídicas avaliadas neste estudo, na faixa etária de 12 a 14 anos.

No sexo feminino, o Índice $\mathrm{C}$ teve um maior poder preditivo na faixa etária de 10 a 11 anos, no entanto somente foi bom preditor para alterações no LDL na faixa etária de 12 a 14 anos. Esse resultado levanta, dessa forma, algumas hipóteses: (i) apesar de as adolescentes do sexo feminino, na faixa etária de 12 a 14 anos, apresentarem uma tendência de acumular gordura, ela é distribuída para glúteos, mamas, quadris e região abdominal 43,44,45; (ii) mesmo sendo difícil atribuir uma idade com precisão, estima-se que valores plenos de massa livre de gordura sejam atingidos nas adolescentes do sexo feminino em torno dos 14 a 16 anos de idade 47,48; e (iii) é possível que seja uma característica da amostra e da população estudada.

Os pontos de corte encontrados neste estudo mostram que, quando o adolescente estiver acima dos valores estabelecidos, para cada faixa etária e de acordo com o sexo, o mesmo terá maiores chances de apresentar alguma alteração lipídica, seja ela hipercolesterolemia, hipertrigliceridedemia, hipoalfaliproteinemia ou LDL-elevado.

Até o momento, só foi localizado um estudo 17 que propusesse um ponto de corte do Índice C para predizer alterações no perfil lipídico em adolescentes, porém o estudo avaliou apenas colesterol total elevado e HDL baixo e não estratificou por faixa etária. Além disso, os pontos de corte estabelecidos foram arredondados para 1,1 em ambas as alterações.

Atualmente, o padrão dislipidêmico na infância e adolescência é caracterizado por elevação (moderada a grave) de triglicerídeos, ligeira alteração no LDL-c e redução do HDL-c 40. Esse perfil lipídico é considerado aterogênico e está associado com a obesidade em crianças e adolescentes 40 . Nesse sentido, o Índice C pode vir a ser utilizado na triagem dessas alterações no perfil lipídico de adolescentes, apesar de os valores de sensibilidade e especificidade não serem muito elevados. Neste estudo, em adolescentes do sexo masculino, a sensibilidade para essas alterações lipídicas foi de aproximadamente $65 \%$ e a especificidade de $63 \%$, assim como em adolescentes do sexo feminino cuja sensibilidade foi de aproximadamente $61 \%$ e especificidade de $59 \%$. Assim, a possibilidade de classificações incorretas é maior entre aqueles com perfil lipídico normal, gerando maior quantidade de falsos positivos. Em se tratando de teste diagnóstico, essa possibilidade não apresenta problemas, visto que teremos menor quantidade de pessoas classificadas como falsos negativos (teste com maior sensibilidade do que especificidade).

Uma limitação para o uso do Índice $\mathrm{C}$ em estudos populacionais é a dificuldade de se calcular o denominador da equação proposta para sua determinação 50. Apesar de a rede básica de saúde no Brasil já ser informatizada e realizar medidas de peso e estatura, sabe-se que, na prática, torna-se difícil o cálculo do denominador do Índice C. Pensando nisso, este estudo elaborou uma tabela, levando em consideração a média e desvio-padrão do peso e estatura da amostra, semelhante à desenvolvida em outro estudo com adultos 50. Pelo cruzamento das informações de peso e estatura é possível verificar o valor do denominador do Índice C já calculado para adolescentes (Tabela 4). Para determinar o Índice 
C, é preciso apenas dividir o valor da circunferência da cintura (em metros) pelo valor do denominador apresentado na Tabela 440.

Para adolescentes com peso superior a $50 \mathrm{~kg}$ e estatura acima de $1,50 \mathrm{~m}$, os valores propostos para os adultos poderão ser utilizados, tendo em vista para o cálculo do denominador do Índice $\mathrm{C}$ o que importa são as medidas de peso e estatura.

\section{Conclusão}

O Índice C pode ser utilizado para predizer alterações lipídicas, bem como seus pontos de corte podem ser utilizados para triagem de adolescentes na identificação do risco de alterações no perfil lipídico. Todavia, ainda são necessários outros estudos que comparem o poder preditivo do Índice C com outros indicadores, em adolescentes, estratificados por sexo e idade.

\section{Colaboradores}

A. C. P. Arruda Neta desenvolveu o estudo, coletou e analisou os dados e construiu o artigo. J. C. Farias Júnior contribuiu com as análises estatísticas e o desenvolvimento do estudo. P. R. Martins contribuiu com o desenvolvimento do artigo, além das análises dos resultados. F. E. L. L. Ferreira contribuiu com o desenvolvimento do artigo, além de auxiliar nas análises estatísticas.

\section{Agradecimentos}

Agradecimentos à Fundação de Apoio à Pesquisa da Paraíba (FAPESQPB) e ao Conselho Nacional de Desenvolvimento Científico e Tecnológico (CNPq) pelo patrocínio e viabilização da pesquisa. Agradecimentos aos adolescentes que compõem o estudo LONCAAFS. 


\section{Referências}

1. Dixon JB, O'Brien PE. Lipid profile in the severely obese: changes with weight loss after lap-band-survery. Obes Res Clin Pract 2002; 10:903-10.

2. Lima SCVC, Arrais RF, Almeida MG, Souza ZM, Pedrosa LFC. Perfil lipídico e peroxidação de lipídeos no plasma de crianças e adolescentes com sobrepeso e obesidade. J Pediatr (Rio J.) 2004; 80:228-39.

3. Coronelli CLS, Moura EC. Hipercolesterolemia em escolares e seus fatores de risco. Rev Saúde Pública 2003; 37:24-31

4. Grillo LP, Crispim SP, Siebert NA, Andrade ATW, Rossi A, Campos IC. Perfil lipídico e obesidade em escolares de baixa renda. Rev Bras Epidemiol 2005; 8:75-81.

5. Faria EC, Dalpino F, Takata R. Lípides e liproteínas séricas em crianças e adolescentes ambulatoriais de um hospital universitário público. Rev Paul Pediatr 2008; 26:54-8.

6. Ribeiro RQC, Lotufo PA, Lamounier JA, Oliveira RG, Soares JF, Botter DA. Fatores adicionais de risco cardiovascular associados ao excesso de peso em crianças e adolescentes. $\mathrm{O}$ estudo do coração de Belo Horizonte. Arq Bras Cardiol 2006; 86:408-18.

7. Carvalho DF, Paiva AA, Melo ASO, Ramos AT, Medeiros JS, Medeiros CCM, et al. Perfil lipídico e estado nutricional de adolescentes. Rev Bras Epidemiol 2007; 10:491-8.

8. Suárez NP, Prin MC, Luciani SL, Pilottó MT, Dri MD, Politti IR. Prevalencia de factores de riesgo de enfermedad cardiovascular: obesidad y perfil lipídico. An Pediatr 2008; 68:257-63.

9. Sant'Anna MSL, Tinôco ALA, Rosado LEFPL, Sant'ana LFR, Brito ISS, Araújo LF, et al. Effectiveness of the conicity index and waist to height ratio to predict the percentage of body fat in children. Nutrire 2010; 35:67-80.

10. Valdez R. A simple model-based index of abdominal adiposity. J Clin Epidemiol 1991; 44:955-6.

11. Coniglio RI, Colombo O, Vasquez L, Salgueiro AM, Otero JC, Malaspina MM. Relación entre el indice de conicidad y los factores de riesgo lipoproteicos para la ateroesclerosis coronaria. Medicina 1997; 57:21-8.

12. Greenlund KJ, Valdèz R, Casper ML, Rith-Najarian S, Croft JB. Prevalence and correlates of the insulin resistance syndrome among native americans: The Inter-Tribal Heart Project. Diabetes Care 1999; 22:441-7.

13. Yasmin, Mascie-Taylor CG. Adiposity indices and their relationship with some risk factors of coronary heart disease in middle-age Cambridge men and women. Ann Hum Biol 2000; 27:239-48.

14. Pitanga FJG, Lessa I, Araújo MJ, Magalhães L. Associação entre índice de conicidade e níveis de glicose plasmática. In: Resumos do 5o Congresso Brasileiro de Epidemiologia. Curitiba: Abrasco; 2002. p. 360.
15. Pitanga FJG, Lessa I. Associação entre índice de conicidade e hipertensão arterial sistólica em adultos na cidade de Salvador-BA. In: Resumos do XIV Congresso de Cardiologia do Estado da Bahia. Salvador: Sociedade Brasileira de Cardiologia; 2002. p. 25.

16. Ghosh A, Fitzgerald MH, Bose K, Chaudhuri AB. Association of food patterns, central obesity measure and metabolic risk factors for coronary heart disease (CHD) in middle age Bengalee Hindu men, Calcutta, India. Asia Pac J Clin Nutr 2003; 12:166-71.

17. Beck CC, Lopes AS, Pitanga FJG. Indicadores antropométricos de sobrepeso e obesidade como preditores de alterações lipídicas em adolescentes. Rev Paul Pediatr 2011; 29:46-53.

18. Ellis KJ, Shypailo RJ, Abrams SA, Wong WW. The reference child and adolescent models of body composition. A contemporary comparison. Ann N Y Acad Sci 2000; 904:374-82.

19. World Health Organization. Nutrition in adolescence-issues and challenges for the health sector. Issues in adolescent health and development. Geneva: World Health Organization; 2005.

20. Sociedade Brasileira de Cardiologia. V diretriz brasileira de dislipidemias e prevenção da aterosclerose. Arq Bras Cardiol 2013; 101(4 Suppl 1): $1-22$.

21. Erdreich LS, Lee ET. Use of relative operating characteristic analysis in epidemiology. A method for dealing with subjective judgement. Am J Epidemiol 1981; 114:649-62.

22. Rocha LCS. Avaliação de modelos usados para estimar a probabilidade de default [Dissertação de Mestrado]. Brasília: Universidade Católica de Brasília; 2012.

23. Schisterman EF, Faraggi D, Reiser B, Trevisan M. Statistical inference for the area under the receiver operating characteristic curve in the presence of random measurement error. Am J Epidemiol 2001; 154:174-9.

24. Vaz JCL. Regiões de incerteza para Curva ROC em teste diagnóstico [Dissertação de Mestrado]. São Carlos: Universidade Federal de São Carlos; 2009.

25. Pitanga FJ, Lessa I. Anthropometric indexes of obesity as an instrument of screening for high coronary risk in adults in the city of SalvadorBahia. Arq Bras Cardiol 2005; 85:26-31.

26. Ghosh JR, Bandyopadhyay AR. Comparative evaluation of obesity measures: relationship with blood pressures and hypertension. Singapore Med J 2007; 48:232-5.

27. Pitanga FJG, Lessa I. Indicadores antropométricos de obesidade como instrumento de triagem para risco coronariano em mulheres. Rev Bras Cineantropom Desempenho Hum 2006; 8:14-21. 
28. Moreira SR, Ferreira AP, Lima RM, Arsa G, Campbell CSG, Simões HG, et al. Predicting insulin resistance in children: anthropometric and metabolic indicators. J Pediatr (Rio J.) 2008; 84:47-52.

29. Lee S, Bacha F, Arslanian SA. Waist circumference, blood pressure, and lipid components of the metabolic syndrome. J Pediatr 2006; 149:809-16.

30. Perez B, Landaeta-Jimenez M, Vásquez M. Distribución de la adiposidad en adolescentes mediante el indice de conicidad. Acta Cient Venez 2000; 51:244-51.

31. Porkka KVK, Viikari JSA, Taimela S, Dahl M, Akerblom HK. Tracking and predictiveness of serum lipid and lipoprotein measurements in childhood: a 12-year follow-up. The Cardiovascular Risk in Young Finns Study. Am J Epidemiol 1994; 140:1096-110.

32. Li S, Chen W, Srinivasan SR, Bond MG, Tang R, Urbina EM, et al. Childhood cardiovascular risk factors and carotid vascular changes in adulthood: the Bogalusa Heart Study. JAMA 2003; 290:2271-6.

33. Moura EC, Castro CM, Mellin AS, Figueiredo DB. Perfil lipídico em escolares de Campinas, SP, Brasil. Rev Saúde Pública 2000; 34:499-505.

34. Giuliano ICB, Coutinho MSSA, Freitas SFT, Pires MMS, Zunino JN, Ribeiro RQC. Lípides séricos em crianças e adolescentes de Florianópolis, SC-Estudo Floripa Saudável 2040. Arq Bras Cardiol 2005; 85:85-91.

35. Kit BK, Kuklina E, Carroll MD, Ostchega Y, Freedman DS, Ogden CL. Prevalence of and trends in dyslipidemia and blood pressure among US children and adolescents, 1999. 2012. JAMA Pediatr 2015; 169:272-9.

36. National Cholesterol Education Program (NCEP) Expert Panel on Detection, Evaluation, and Treatment of High Blood Cholesterol in Adults (Adult Treatment Panel III). Third report of the National Cholesterol Education Program (NCEP). Expert Panel on Detection, Evaluation, and Treatment of High Blood Cholesterol in Adults (Adult Treatment Panel III) final report. Circulation 2002; 106:3143-421.

37. Faria-Neto JR, Bento VFR, Baena CP, Olandoski M, Gonçalves LGO, Abrau GA, et al. ERICA: prevalência de dislipidemia em adolescentes brasileiros. Rev Saúde Pública 2016; 50 Suppl $1: 10$ s.
38. Pereira PB, Arruda IKG, Cavalcanti AMTS, Diniz AS. Perfil lipídico em escolares de Recife PE. Arq Bras Cardiol 2010; 95:606-13.

39. Cook S, Kavey REW. Dyslipidemia and pediatric obesity. Pediatr Clin North Am 2011; 58:1363-73.

40. Kavey RE. Combined dyslipidemia in childhood. J Clin Lipidol 2015; 9(5 Suppl):S41-56.

41. Stamler J, Daviglus ML, Garside DB, Dyer AR, Greenland P, Neaton JD. Relationship of baseline serum cholesterol levels in 3 large cohorts of younger men to long-term coronary, cardiovascular, and all-cause mortality and to longevity. JAMA 2000; 284:311-8.

42. Daniels SR, Morrison JA, Sprecher DL, Khoury P, Kimball TR. Association of body fat distributon and cardiovascular risk factors in children and adolescents. Circulation 1999; 99:541-5.

43. Daniels SR, Arnett DK, Eckel RH, Gidding SS, Hayman LL, Kumanyika S, et al. Overweight in children and adolescents: pathophysiology, consequences, prevention, and treatment. Circulation 2005; 111:1999-2012.

44. Rogol AD. Sex steroids, growth hormone, leptin and the pubertal growth spurt. Endocr Dev 2010; 17:77-85.

45. Rogol AD, Roemmich JN, Clark PA. Growth at puberty. J Adolescent Health 2002; 31:192-200.

46. Ellis KJ, Shypailo RJ, Abrams SA, Wong WW. The reference child and adolescent models of body composition. A contemporary comparison. Ann N Y Acad Sci 2000; 904:374-82.

47. Malina RM, Bouchard C, Bar-Or O. Crescimento, maturação e atividade física. São Paulo: Phorte; 2009.

48. Tommiska J, Wehkalampi K, Vaaralahti K, Laitinen EM, Raivio T, Dunkel L. Lin28b in constitutional delay of growth and puberty. J Clin Endocrinol Metab 2010; 95:3063-6.

49. Jolliffe CJ, Janssen I. Distribution of lipoproteins by age and gender in adolescents. Circulation 2006; 114:1056-62.

50. Pitanga FJG, Lessa I. Sensibilidade e especificidade do índice de conicidade como discriminador do risco coronariano de adultos em Salvador, Brasil. Rev Bras Epidemiol 2004; 7:259-69. 


\section{Abstract}

The objective was to evaluate the conicity index (C Index) as a predictor of changes in the lipid profile of adolescents and to establish its cutoff points. This was a cross-sectional study in 774 adolescents of both sexes (55\% girls), 10 to 14 years of age. $C$ Index was calculated according to the formula proposed by Valdez, considering body mass, height, and waist circumference (WC). Changes in the adolescents' lipid profile were defined according to one of the following conditions: elevated levels of total cholesterol, low-density lipoprotein ( $L D L)$, and triglycerides and low levels of high-density lipoprotein (HDL). The predictive power of the conicity index for altered lipid profile and its cutoff points were determined by Receiver Operating Characteristic (ROC) curves. The C Index was a good predictor of lipid alterations in adolescents, emphasizing triglycerides in boys 10 to 11 years of age $(R O C=0.67 ; 95 \% \mathrm{CI}: 0.50-0.85)$ and 12 to 14 $(R O C=0.69 ; 95 \% C I: 0.59-0.80)$, and in girls 10 to 11 years $(R O C=0.65 ; 95 \% \mathrm{CI}: 0.50-0.79)$; and $L D L$ in girls 10 to 11 years $(R O C=0.70 ; 95 \% \mathrm{CI}$ : $0.59-0.80)$ and boys $(R O C=0.65$; 95\% CI: 0.55 $0.75)$ and girls $(R O C=0.62 ; 95 \%$ CI: $0.50-0.75)$ 12 to 14 years. The cutoff points for the $C$ Index varied from 1.12 to 1.16 between boys and girls. The C Index can be used to predict lipid alterations, and its cutoff points can be used to screen adolescents for risk of alterations in lipid profile.

Dyslipidemias; Abdominal Obesity; Waist Circumference; Adolescent

\section{Resumen}

El objetivo fue evaluar el índice de conicidad (Índice C), como predictor de alteraciones en el perfil lipídico en adolescentes y establecer sus puntos de corte. Métodos: estudio transversal con 774 adolescentes de ambos sexos (55\% del sexo femenino), de 10 a 14 años de edad. El Índice C fue calculado de acuerdo con la fórmula propuesta por Valdez, considerando las medidas de masa corporal, estatura $y$ circunferencia de la cintura (CC). Las alteraciones en el perfil lipídico de los adolescentes fueron definidas según una de las siguientes condiciones: elevados niveles de colesterol total, lipoproteína de alta densidad ( $L D L)$, triglicéridos y niveles bajos de lipoproteina de baja densidad (HDL). El poder predictivo del Índice $C$ para alteraciones en el perfil lipídico, así como sus puntos de corte, fue determinado mediante las curvas ROC (Receiver Operating Characteristic). El Índice C fue un buen predictor para alteraciones lipídicas en adolescentes, destacando los niveles de triglicéridos en niños de 10 a 11 años (ROC = 0,67; IC95\%: 0,50-0,85) y de 12 a 14 años $(R O C=0,69$; IC95\%: 0,59-0,80), así como en niñas de 10 a 11 años $(R O C=0,65 ;$ IC95\%: 0,50-0,79); $y$ LDL en niñas de 10 a 11 años $($ ROC = 0,70; IC95\%: 0,59-0,80) $y$ niños $(R O C=0,65$; IC95\%: 0,55-0,75) y niñas $(R O C=0,62$; IC95\%: 0,50-0,75) de 12 a 14 años. Los puntos de corte del Índice C variaron de 1,12 a 1,16 entre niños y niñas. El Índice C puede ser utilizado para predecir alteraciones lipídicas, así como sus puntos de corte pueden ser utilizados para el cribado de adolescentes en riesgo de alteraciones en su perfil lipídico.

Dislipidemias; Obesidad Abdominal; Circunferencia de la Cintura; Adolescente

Recebido em 21/Fev/2016

Versão final reapresentada em 25/Mai/2016 Aprovado em 13/Jun/2016 